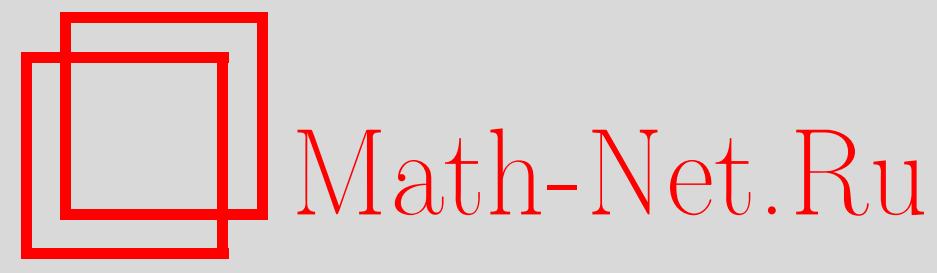

C. K. Ландо, J-инварианты орнаментов и оснащенные хордовые диаграммы, Функи. анализ и его прил., 2006, том 40, выпуск 1, 1-13

DOI: https://doi.org/10.4213/faa14

Использование Общероссийского математического портала Math-Net.Ru подразумевает, что вы прочитали и согласны с пользовательским соглашением

http://www . mathnet.ru/rus/agreement

Параметры загрузки:

IP : 34.239 .49 .27

26 апреля 2023 г., 17:20:46

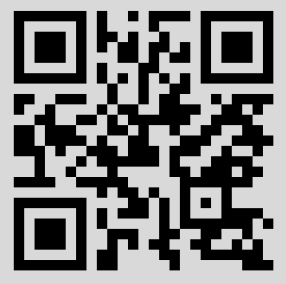


Функииональный анализ и его приложения

2006, т. 40, вып. 1, с. 1-13

УДК 515.1

\title{
$J$-инварианты орнаментов и оснащенные хордовые диаграммы*
}

\author{
(C) 2006. С. К. ЛАНдо
}

Принадлежащая Васильеву [19] теория инвариантов конечного порядка узлов тесно связана с рассматривавшейся Арнольдом $[1,2]$ теорией $J$-инвариантов конечного порядка плоских кривых. В этом нет ничего удивительного, поскольку такая кривая представляет собой проекцию на плоскость лежандрова узла в проективизированном касательном расслоении к этой плоскости, а $J$-инварианты, отвечающие за перестройки плоских кривых при самокасании, соответствуют инвариантам Васильева, отвечающим за перестройки лежандровых узлов при прохождении одной ветви узла через другую. Горюнов [7] дал полное описание $J^{+}$-инвариантов конечного порядка (отвечающих за перестройки при попутном самокасании) в терминах помеченных хордовых диаграмм, аналогичных обычным хордовым диаграммам, в терминах которых описываются инварианты Васильева узлов. В описании Горюнова всякий $J^{+}$-инвариант конечного порядка определяет функцию на помеченных хордовых диаграммах, подчиняющуюся так называемым помеченным 4-членным соотношениям. Он отметил также, что и $J^{-}$-инварианты (отвечающие за перестройку противопутного самокасания) допускают подобное описание. Соответствующие соотношения были выписаны в [8] без перевода на язык хордовых диаграмм.

Вместе с тем до сих пор $J^{+}$- и $J^{-}$-инварианты существовали независимо друг от друга. Цель настоящей работы - объединить $J^{+}-$и $J^{-}$-инварианты в единый класс $J$-инвариантов. Для описания инвариантов конечного порядка этого класса используется единый комбинаторный объект, оснащенная хордовая диаграмма, сопоставляемая плоской кривой, имеющей особенности как $J^{+}$, так и $J^{-}$-типов. При этом каждая хорда диаграммы, сопоставляемая точке самокасания кривой, получает оснащение - элемент кольца вычетов по модулю 2 , равный 0 или 1 в зависимости от того, является ли самокасание попутным или противопутным. Несмотря на то что понятие оснащения хордовой диаграммы появлялось, скажем, в [14] и при обсуждении so $(N)$-инвариантов в [4], его самостоятельное значение, похоже, до сих пор не было осознано. Функции на хордовых диаграммах, которые строятся по $J$-инвариантам конечного порядка, должны удовлетворять оснащенным 4-членным соотношениям, обобщающим классические 4-членные соотношения Васильева. Описанию оснащенных хордовых диаграмм, 4-членных соотношений для них и их связи с $J$-инвариантами посвящены первые два параграфа статьи. Отметим, что пространство оснащенных хордовых диаграмм по модулю 4-членных соотношений наделяется естественной структурой градуированной кокоммутативной коалгебры, а вот во-

* Работа выполнена при поддержке грантов ACI-NIM-2004-243 (Noeuds et tresses), HBOРФФИ 047.011.2004.026 (РФФИ 05-02-89000-НВО-а), РФФИ 02-01-22004-НЦНИ-а, РФФИ 0501-01012-а и GIMP ANR-05-BLAN-0029-01. 
прос о существовании в нем естественного умножения (и превращения его тем самым в биалгебру) остается открытым.

У биалгебры обычных хордовых диаграмм есть графовый аналог - 4-биалгебра (алгебра Хопфа) графов, введенная в [12]. Оснащенные хордовые диаграммы также имеют аналог - оснащенные графы. Под оснащенным графом мы понимаем простой граф, каждой вершине которого приписан элемент кольца вычетов по модулю 2. Для оснащенных графов можно написать оснащенное 4-членное соотношение, обобщающее 4-членное соотношение для обычных графов из [12]. Умножение оснащенных графов задается несвязным объединением графов, и вопрос о корректности его определения попросту не встает. Важность введения алгебры Хопфа оснащенных графов и необходимость ее изучения объясняется существованием гомоморфизма из коалгебры оснащенных хордовых диаграмм в алгебру Хопфа оснащенных графов (а значит, обратного гомоморфизма инвариантов). Алгебра Хопфа оснащенных хордовых диаграмм обсуждается в третьем параграфе. Отметим, что оснащенные хордовые диаграммы и оснащенные графы рассматривались в [14] (там они называются помеченными хордовыми диаграммами и графами, но их не надо путать с упоминавшимися выше помеченными хордовыми диаграммами по Горюнову) именно в связи с весовыми системами. Однако в этой статье для них не вводятся ни 4-членное соотношение, ни соответствующие алгебраические структуры.

Четвертый параграф посвящен обсуждению возможной комплексификации $J$-инвариантов конечного порядка. Он инспирирован тем наблюдением, что у оснащенной хордовой диаграммы есть весьма естественный комплексный аналог - оснащенное зацепление в $S^{3}$. Другие детали комбинаторного описания также переносятся на комплексный случай. Если понимать плоские (точнее, сферические) кривые как следы пересечения двумерных поверхностей с единичной сферой в трехмерном пространстве, то их комплексным аналогом должны служить пересечения двумерных комплексных поверхностей в $\mathbb{C}^{3}$ с единичной (пятимерной) сферой. Самокасания таких поверхностей и описываются комплексными хордовыми диаграммами.

Я благодарен С. Дужину, М. Капранову и М. Поляку за проявленный интерес к работе. На начальной стадии работы чрезвычайно плодотворными оказались обсуждения с А. Левиным. Я также благодарен С. Чмутову, указавшему мне на статью Горюнова [7], и неизвестному рецензенту, указавшему на статью Хилла [8]. Часть работы была выполнена во время моего пребывания в Институте математики Макса Планка в Бонне в апреле-июле 2001 года, и я благодарен институту за гостеприимство.

\section{§1. Оснащенные хордовые диаграммы}

Основным комбинаторным объектом в нашем исследовании является оснащенная хордовая диаграмма. По определению оснащенная хордовая диаграмма - это неориентированная хордовая диаграмма порядка $n$ (т. е. окружность, на которой заданы $n$ пар попарно различных точек - хорд, рассматриваемая с точностью до не обязательно сохраняющих ориентацию диффеоморфизмов) с заданным на ней оснащением. Под оснащением мы понимаем отображение, сопоставляющее каждой хорде хордовой диаграммы элемент кольца $\mathbb{Z} / 2 \mathbb{Z}$ вычетов по модулю 2, см. рис. 1а). Хорды с оснащением 0 будем называть ориентируемыми, а хорды с оснащением 1 - дезориентирующими или скрученными. 
В принципе, наряду с неориентированными оснащенными хордовыми диаграммами можно рассматривать и ориентированные. До введения умножения в модуле оснащенных хордовых диаграмм (см. ниже) неясно, какой из этих двух подходов предпочтительнее

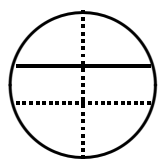

a)

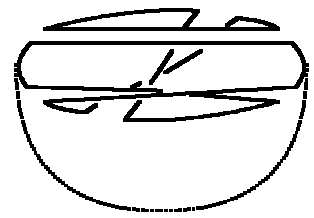

b)

Рис. 1. а) Оснащенная хордовая диаграмма $c$ и b) сопоставляемая ей поверхность $S(c)$. Ориентируемая хорда диаграммы изображена непрерывной линией, а дезориентирующие хорды - пунктиром

Каждой оснащенной хордовой диаграмме $c$ можно следующим образом сопоставить двумерную поверхность $S(c)$ с краем. Приклеим к несущей окружности диаграммы $c$ диск, а каждую хорду превратим в ручку индекса 1, не скручивая, если ее оснащение равно 0, и скрутив один раз, если ее оснащение равно 1. Пример построения такой поверхности по заданной оснащенной хордовой диаграмме изображен на рис. 1b). Очевидно, что поверхность $S(c)$ ориентируема в том и только в том случае, если оснащение всех хорд равно 0.

Как и в теории инвариантов Васильева (см. [4]), мы можем рассмотреть модуль над произвольным коммутативным ассоциативным кольцом с единицей $($ скажем, над $\mathbb{Z})$, порожденный хордовыми диаграммами и профакторизованный по 4-членным соотношениям, изображенным на рис. 2 , 3 . На всех рисунках дуги окружности, нарисованные непрерывной линией, содержат лишь концы изображенных на этих рисунках хорд хордовых диаграмм, тогда как пунктирные дуги могут содержать концы произвольного набора хорд - одного и того же для всех четырех членов соотношения. Такое 4-членное соотношение задается хордовой диаграммой $c$ и парой $A, B$ ее хорд, имеющих соседние концы. Как и всюду на рисунках, хорды с оснащением 0 изображаются непрерывной линией, а хорды с оснащением 1 - пунктиром.

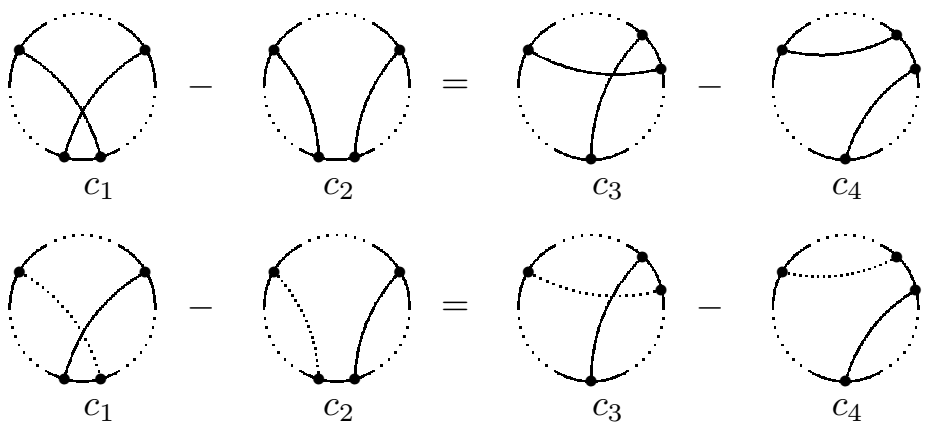

Рис. 2. Оснащенные 4-членные соотношения; ориентируемый случай 


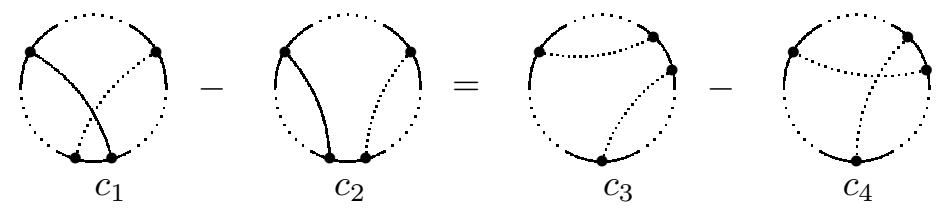

Рис. 3. Оснащенное 4-членное соотношение; дезориентирующий случай

Переход от диаграммы $c_{1}$ к диаграмме $c_{2}$ (или от $c_{3}$ к $c_{4}$ ) в 4-членном соотношении описать просто: он состоит в перестановке соседних концов двух хорд, задающих соотношение. Мы называем эту операцию первым двиюением Васильева. Переход от $c_{1}$ к $c_{3}$ (или от $c_{2}$ к $c_{4}$ ) менее тривиален. На языке поверхности $S(c)$ он состоит в протягивании ручки $A$, сопоставляемой хорде $A$, вдоль ручки $B$, сопоставляемой хорде $B$, см. рис. 4 . Мы называем это протягивание вторым движсением Васильева. Интерпретация этого движения в терминах поверхности $S(c)$ объясняет выбор положения второго конца и оснащения модифицированной хорды $A$ после протягивания вдоль хорды $B$. Отметим, что если все хорды диаграммы имеют оснащение 0, то 4-членное соотношение для любой пары из них совпадает с обычным.

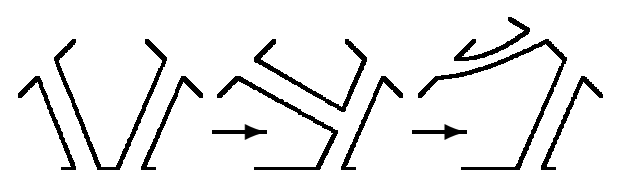

a)

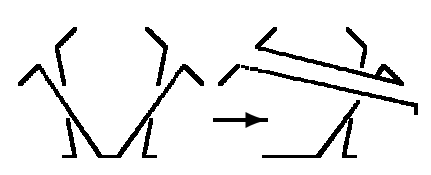

b)

Рис. 4. Второе движение Васильева как протягивание ручки.

Ориентируемый, a), и дезориентирующий, b), случаи

Обозначим фактормодуль хордовых диаграмм порядка $n$ по всем 4-членным соотношениям через $\mathscr{M}_{n}^{f}$. Мы полагаем $\mathscr{M}_{0}^{f}$, модуль, порожденный пустой хордовой диаграммой, равным $\mathbb{Z}$. Прямая сумма

$$
\mathscr{M}^{f}=\mathscr{M}_{0}^{f} \oplus \mathscr{M}_{1}^{f} \oplus \cdots
$$

наделяется естественной структурой градуированной коалгебры. А именно, определим градуированное коумножение

$\mu: \mathscr{M}^{f} \rightarrow \mathscr{M}^{f} \otimes \mathscr{M}^{f}, \quad \mu: \mathscr{M}_{n}^{f} \rightarrow \mathscr{M}_{0}^{f} \otimes \mathscr{M}_{n}^{f}+\mathscr{M}_{1}^{f} \otimes \mathscr{M}_{n-1}^{f}+\cdots+\mathscr{M}_{n}^{f} \otimes \mathscr{M}_{0}^{f}$, обычным образом; это означает, что

$$
\mu: c \mapsto \sum c_{I} \otimes c_{J}
$$

где суммирование ведется по всем упорядоченным разбиениям множества хорд диаграммы $c$ на два непересекающихся подмножества $I$ и $J$, а через $c_{I}$ обозначена поддиаграмма диаграммы $c$, образованная хордами из подмножества $I$. На линейные комбинации диаграмм коумножение продолжается по линейности.

ТЕОрема 1.1. Определенное выше коумножение $\mu$ вводит на модуле оснащенных хордовых диаграмм $\mathscr{M}^{f}$ структуру градуированной коассоциативной кокоммутативной коалгебры.

ДокАзАтЕльство. Доказательство теоремы стандартно. Мы должны проверить, что результат применения коумножения к 4-членному соотношению равен 
нулю по модулю 4-членных соотношений в тензорном квадрате $\mathscr{M}^{f} \otimes \mathscr{M}^{f}$. Воспользуемся тем, что множества хорд во всех четырех членах любого 4-членного соотношения попарно отождествлены друг с другом. Возьмем произвольное слагаемое $c_{I} \otimes c_{J}$ в копроизведении первого члена соотношения. Тогда возможны два случая: либо обе хорды $A, B$, задающие соотношение, лежат в одном подмножестве (скажем, $I$ ), либо они лежат в разных подмножествах. В первом случае объединим все 4 члена, которые строятся по диаграмме $c_{I}$ и хордам $A$ и $B$ в ней, и получим произведение 4-членного соотношения на $c_{J}$. Во втором случае достаточно разбить слагаемые в копроизведении на пары, соответствующие левой и правой частям 4-членного соотношения - каждая из пар равна нулю сама по себе. Кокоммутативность и коассоциативность коумножения очевидны.

ЗАмЕчАНИЕ 1.2. В модуле обычных хордовых диаграмм помимо коумножения имеется также естественное коммутативное умножение, согласованное с коумножением и превращающее тем самым модуль в биалгебру (а значит, и в алгебру Хопфа). Это умножение определяется следующим образом: возьмем две хордовые диаграммы $c_{1}, c_{2}$, разорвем обе окружности, на которых они заданы, в произвольной точке, отличной от концов хорд, и склеим эти две разорванные окружности в одну с сохранением ориентации. Для доказательства корректности определения необходимо показать, что результат умножения не зависит от выбора точек разрыва на каждой из окружностей. По модулю 4-членных соотношений это действительно так. Естественное желание распространить это определение на оснащенные хордовые диаграммы пока не реализовано - мне не удается показать, что такое определение корректно. Можно, конечно, определить произведение как симметризованную линейную комбинацию диаграмм, просуммированных по всем возможным выборам точек разрыва, однако такой подход выглядит трудоемким и не вполне оправданным.

Определим оснащенную весовую систему порядка $n$ как линейный функционал на $\mathscr{M}_{n}^{f}$. Другими словами, это функция на оснащенных хордовых диаграммах, удовлетворяющая 4-членным соотношениям, т. е. такая, что результаты подстановки в нее всех четырех диаграмм с каждого из рис. 2, 3 удовлетворяют тем же соотношениям, что и сами диаграммы. Примером такой функции является число связных компонент границы поверхности $S(c)$. Ее значение на оснащенной хордовой диаграмме с рис. 1 равно 1. Проверка 4-членного соотношения очевидна. Действительно, эта весовая система удовлетворяет даже 2-членному соотношению - ее значение на оснащенной хордовой диаграмме не меняется при втором движении Васильева, поскольку это второе движение сохраняет топологию поверхности $S(c)$.

\section{§2. J-инварианты плоских кривых}

Наши определения инвариантов плоских кривых следуют Арнольду [1,2]. Плоской уникурсальной кривой называется гладкое отображение окружности $S^{1}$ в плоскость $\mathbb{R}^{2}$. Такое отображение неособо, если оно является невырожденным в каждой точке окружности, а его образ имеет лишь простые (двойные) самопересечения. Две такие кривые эквивалентны, если одна из них переходит в другую при некоторой изотопии плоскости, содержащей кривую, и (не обязательно сохраняющем ориентацию) диффеоморфизме отображаемой окружности. Инвариантом плоских кривых будем называть функцию на неособых 
кривых, принимающую одинаковые значения на эквивалентных кривых. Разумеется, можно рассматривать инварианты кривых и на поверхностях, отличных от плоскости.

В однопараметрических семействах плоских кривых неустранимым образом встречаются кривые с особенностями. Все глобальные неустранимые особенности делятся на два типа:

- три ветви образа попарно трансверсально пересекаются в одной точке (особенность типа странности). Проход через такую особенность не меняет числа точек самопересечения кривой;

- две ветви кривой касаются друг друга в изолированной точке. При перестройке такого вида происходит рождение либо уничтожение пары двойных точек. Эти особенности разбиваются на два подкласса: прямое (попутное) самокасание (если касательные векторы ветвей в точке самокасания сонаправлены; особенности этого подкласса называются $J^{+}$-особенностями) и обратное (противопутное) самокасание (если касательные векторы ветвей в точке касания имеют противоположные направления; эти особенности называются $J^{-}$-особенностями), см. рис. 5.
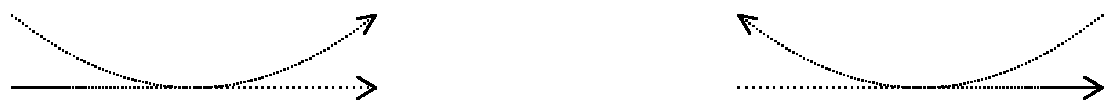

Рис. 5. Прямое (слева) и обратное (справа) самокасания

Отметим, что для определения характера самокасания (прямое или обратное) необходимо ориентировать отображаемую окружность, однако результат не зависит от выбора ориентации, что и позволяет нам работать с неориентированными окружностями.

Ниже нас будут интересовать лишь инварианты типа $J$, т. е. такие, значения которых меняются при проходе через кривые с точками самокасания, и мы не будем обращать внимания на инварианты типа странности.

Будем называть особой кривой гладкое невырожденное отображение окружности в плоскость, имеющее точки простого самокасания и, быть может, точки простого самопересечения и не имеющее других особенностей. Особые кривые образуют дискриминант в пространстве кривых. Эквивалентность для особых кривых определяется так же, как и для неособых.

Число $n$ точек самокасания особой кривой является инвариантом эквивалентности; мы будем называть его порядком особой кривой. Особой кривой порядка $n$ можно следующим образом сопоставить оснащенную хордовую диаграмму: рассмотрим окружность-прообраз, на которой выделены пары прообразов точек самокасания особой кривой. При этом оснащение хорды равно 0, если самокасание прямое, и 1 для обратного самокасания. Неособой кривой соответствует пустая хордовая диаграмма. Отметим, что хордовую диаграмму особой кривой не следует путать с гауссовой диаграммой неособой кривой, хордами в которой являются прообразы двойных точек.

Следующее утверждение очевидно.

ПРЕДЛОЖЕНИЕ 2.1. Всякая оснащенная хордовая диаграмма является хордовой диаграммой некоторой особой кривой. 
Инвариант особых кривых называется продолжением инварианта неособых кривых, если его значение на особых кривых подчиняется правилу, изображенному на рис. 6. Это правило означает, что мы можем восстановить значение продолжения инварианта на любой особой кривой порядка $n$ по его значениям на неособых кривых, взяв альтернированную сумму его значений по всем $2^{n}$ неособым разрешениям кривой. Будем называть инвариант неособых кривых $J$-инвариантом, если он не меняется при прохождении кривой с тройной точкой. Ясно, что любой $J$-инвариант допускает продолжение на особые кривые: четверка кривых, получающаяся в результате всех возможных разрешений двух точек самокасания, не зависит от порядка разрешения этих точек.

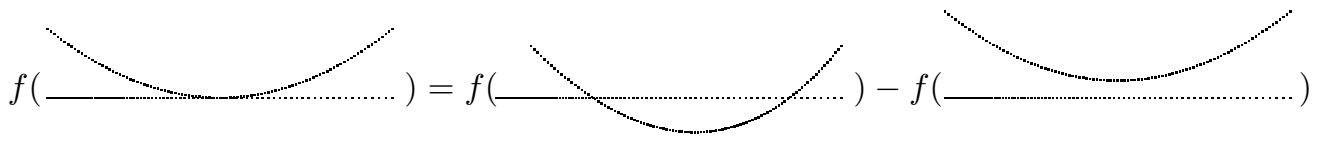

Рис. 6. Правило продолжения $J$-инварианта на дискриминант

$J$-инвариант называется инвариантом порядка не выше $n$, если его значения на всех особых кривых с более чем $n$ точками самокасания равны нулю. Все $J$-инварианты конечного порядка со значениями в данном кольце образуют алгебру $J$-инвариантов Васильева над этим кольцом. Стандартным образом доказывается

ПРЕДЛОЖЕНИЕ 2.2. Значение J-инварианта порядка не выше $n$ на особой кривой с $n$ точками самокасания однозначно определяется оснащенной хордовой диаграммой этой кривой.

Действительно, нетрудно видеть, что любые две особые кривые с одинаковыми оснащенными хордовыми диаграммами переводятся друг в друга с помощью перестроек, каждая из которых представляет собой проход через особую кривую с дополнительной тройной точкой или дополнительной точкой самокасания. Согласно определению, скачок инварианта при такой перестройке равен нулю. Доказанное утверждение означает, что всякому $J$-инварианту порядка не выше $n$ сопоставляется некоторая функция на оснащенных хордовых диаграммах порядка $n$.

Теорема 2.3. Любая функиия на оснащенных хордовых диаграммах порядка $n$, полученная из J-инварианта порядка не выше $n$, удовлетворяет 4-членному соотношению, т. е. является оснащенной весовой системой.

Для случая $J^{+}$-инвариантов (т. е. $J$-инвариантов, испытывающих скачок лишь при прохождении прямых самокасаний) эта теорема доказана в [7], причем способ доказательства совпадает с тем, что описан ниже. Разумеется, там речь идет об обычных, неоснащенных хордовых диаграммах. На самом деле результат из [7] в неоснащенном случае гораздо точнее - там дано полное описание $J^{+}$-инвариантов конечного порядка в терминах так называемых nомеченных хордовых диаграмм (на дугах которых указываются индексы вращения соответствующих им отрезков особой кривой). Аналогичное рассуждение для $J^{-}$-инвариантов - без упоминания хордовых диаграмм - имеется в [8].

ДокАзАтЕльство. Для доказательства достаточно обратиться к рис. 7, описывающему все возможные разрешения «точки тройного самокасания», которая не встречается у наших отображений. Разность первых двух картинок в левой 
части равенств на рис. 7 равна разности вторых двух картинок, взятых с обратным знаком. Перебирая все возможные взаимные ориентации трех ветвей кривой в точке тройного самокасания и переводя изображенные рисунки на язык оснащенных хордовых диаграмм с выделенными хордами $A, B$, мы получаем в точности оснащенные 4-членные соотношения. Отметим, что при вычислении разности первых двух картинок мы пользуемся возможностью двойного прохода через тройную точку, см. рис. 8.
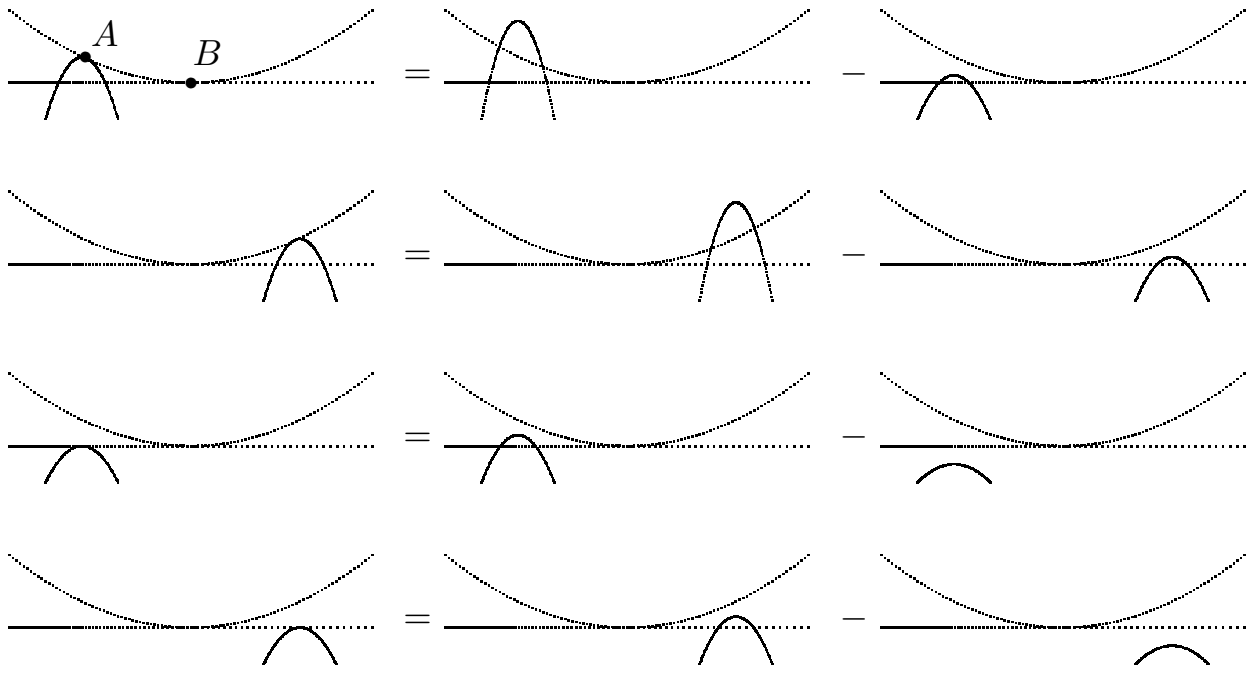

Рис. 7. Вывод 4-членных соотношений
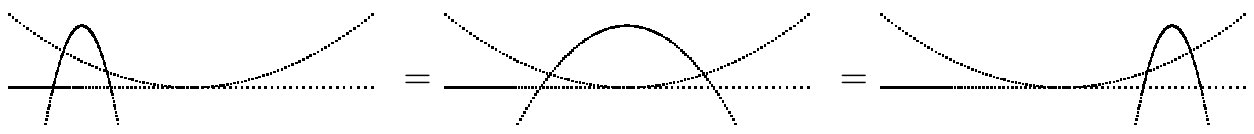

Рис. 8. Двукратный проход через тройную точку

\section{§3. Оснащенные графы}

Понятие оснащения распространяется на графы пересечений хордовых диаграмм. Напомним, что графом пересечения $G(c)$ хордовой диаграммы $c$ называется граф, вершины которого взаимно однозначно соответствуют хордам диаграммы и две вершины соединены ребром, если и только если соответствующие им хорды пересекаются. Оснащенным графом мы называем простой (т. е. неориентированный, не имеющий петель и кратных ребер) граф с оснащениeм, т. е. отображением, сопоставляющим каждой вершине графа элемент кольца $\mathbb{Z} / 2 \mathbb{Z}$ вычетов по модулю 2 . Граф пересечений $\Gamma(c)$ оснащенной хордовой диаграммы $c$ - это оснащенный граф, вершины которого взаимно однозначно соответствуют хордам диаграммы, оснащение каждой вершины равно оснащению соответствующей хорды и две вершины соединены ребром, если и только если соответствующие им хорды пересекаются. 
Зафиксируем какую-нибудь нумерацию числами от 1 до $n$ вершин оснащенного графа $\Gamma$ с $n$ вершинами. Под матрицей примыканий $A(\Gamma)$ графа $\Gamma$ мы понимаем $n \times n$-матрицу с элементами из $\mathbb{Z} / 2 \mathbb{Z}$, в недиагональной клетке $(i, j)$ которой стоит 1 , если вершины $i$ и $j$ соединены ребром, и 0 в противном случае, а диагональные элементы совпадают с оснащением соответствующих вершин.

Для оснащенных графов пересечений оснащенных хордовых диаграмм эта матрица очень тесно связана с топологией поверхности $S(c)$. Она является не чем иным, как матрицей пересечений в первых гомологиях этой поверхности, записанной в следующем базисе. Свяжем с каждой хордой в оснащенной хордовой диаграмме $c$ 1-цикл в $S(c)$, получаемый соединением концов хорды отрезком в диске, приклеенном к окружности при построении поверхности. Такой 1-цикл определяет элемент в $H_{1}(S(c), \mathbb{Z} / 2 \mathbb{Z})$, а все циклы в совокупности задают базис в этом $\mathbb{Z} / 2 \mathbb{Z}$-модуле. Очевидно, что матрица примыканий $A(\Gamma(c))$ и есть матрица пересечений в гомологиях, записанная в выбранном базисе.

Интересно следующее свойство этой матрицы.

Теорема 3.1 [14]. Коранг матрищъ примыканий $A(\Gamma(c))$, построенной по оснащенной хордовой диаграмме $c$, совпадает с уменъшенным на единицу числом компонент границы $\partial S(c)$ поверхности $S(c)$.

Для неоснащенного случая это утверждение было доказано в [5, 15, 17]. В [14] по матрице примыканий оснащенного графа пересечений строятся и более изощренные весовые системы, в том числе соответствующие полиномам Конвея, Кауффмана и HOMFLYPT.

Аналогично случаю оснащенных хордовых диаграмм мы можем рассмотреть 4-членное соотношение для оснащенных графов. Такое 4-членное соотношение определяется графом и упорядоченной парой вершин в нем. Для графа Г и вершин $A, B$ первое движение Васильева $\Gamma \mapsto \Gamma_{A, B}^{\prime}$ меняет примыкание вершин $A, B$ на противоположное, а второе движение Васильева $\Gamma \mapsto \widetilde{\Gamma}_{A B}$ меняет на противоположное примыкание вершины $A$ ко всем вершинам $C$, соединенным с $B$. Если оснащение вершины $B$ равно 1 , то второе движение Васильева меняет на противоположное как оснащение вершины $A$, так и ее примыкание к $B$. Таким образом, 4-членное соотношение для оснащенных графов имеет вид

$$
\Gamma-\Gamma_{A B}^{\prime}=\widetilde{\Gamma}_{A B}-\widetilde{\Gamma}_{A B}^{\prime}
$$

На языке матриц примыканий второе движение Васильева соответствует замене базиса $A, B, C, \ldots$ базисом $A+B, B, C, \ldots$ Действительно, имеем $(A+$ $B, C)=(A, C)+(B, C),(A+B, B)=(A, B)+(B, B),(A+B, A+B)=(A, A)+$ $(B, B)$ в соответствии с приведенным выше определением. В такой интерпретации понятно, почему второе движение Васильева не меняет коранга матрицы пересечений.

Обозначим через $\mathscr{F}_{n}^{f}$ модуль, порожденный оснащенными графами с $n$ вершинами и профакторизованный по 4-членным соотношениям. Тогда прямая сумма

$$
\mathscr{F}^{f}=\mathscr{F}_{0}^{f} \oplus \mathscr{F}_{1}^{f} \oplus \cdots
$$

наделяется естественной структурой градуированной коммутативной кокоммутативной биалгебры (и алгебры Хопфа). Умножение $m: \mathscr{F}^{f} \otimes \mathscr{F}^{f} \rightarrow \mathscr{F}^{f}$ в ней индуцируется несвязным объединением графов, а коумножение задается так 
же, как и для случая (оснащенных) хордовых диаграмм, т. е.

$$
\mu: \Gamma \mapsto \sum \Gamma_{I} \otimes \Gamma_{J}
$$

где через $\Gamma_{I}$ обозначен полный подграф в $Г$, индуцированный подмножеством вершин $I$, а суммирование ведется по всем упорядоченным разбиениям множества вершин на два непересекающихся подмножества $I, J$.

TЕорема 3.2. Определенные выше умножение и коумножение задают в $\mathscr{F} f$ структуру градуированной коммутативной кокоммутативной биалгебры.

Теорема доказывается стандартным образом. Отметим, что, в отличие от случая оснащенных хордовых диаграмм, никаких сомнений в корректности определения умножения оснащенных графов не возникает. Следующее утверждение немедленно вытекает из определений.

Теорема 3.3. Отображение $\Gamma: c \mapsto \Gamma(c)$, сопоставляющее оснащенной хордовой диаграмме ее граф пересечений, продолюсается до градуированного гомоморфизма коалгебр $Г: \mathscr{M}^{f} \rightarrow \mathscr{F}^{f}$.

Элемент $p$ коалгебры с коумножением $\mu$ называется примитивным, если $\mu(p)=1 \otimes p+p \otimes 1$. Согласно структурной теореме Милнора-Мура, коммутативная кокоммутативная биалгебра над полем нулевой характеристики изоморфна биалгебре многочленов от своих примитивных элементов. Поэтому важное значение приобретает подсчет размерностей подпространств примитивных элементов в алгебре Хопфа $\mathscr{F} f$, рассматриваемой над полем характеристики нуль. Для $n=1,2,3$ все эти размерности равны 2 . Отметим, что с ростом $n$ вычислительная сложность задачи подсчета размерности быстро растет.

\section{§4. О возможной комплексификации инвариантов Васильева}

Согласно парадигме комплексификации (см., например, [3]), всякое математическое понятие имеет по крайней мере три реализации - вещественную, комплексную и кватернионную. Теоремы, справедливые для вещественной реализации понятия, естественным образом переносятся на его комплексную и кватернионную реализации, что позволяет одновременно строить три параллельные теории. К сожалению, процесс комплексификации (и кватернионизации) вещественного понятия неоднозначен и неалгоритмизуем и о корректности построения можно говорить лишь по его завершении.

По очевидным причинам теория инвариантов узлов конечного порядка вещественная, и вопрос о построении ее правильного комплексного варианта встал сразу же после того, как она оформилась в отдельную теорию. В одном из возможных подходов комплексным аналогом гладких отображений окружности в трехмерное пространство считаются голоморфные отображения компактной комплексной кривой (например, проективной прямой) в трехмерные комплексные многообразия. В этом подходе окружность интерпретируется как вещественная проективная прямая, и тогда комплексная проективная прямая служит ее естественным комплексным аналогом. Для таких отображений можно строить аналоги индекса зацепления [11] либо изучать отображения цепочки проективных прямых, вводя таким образом мультипликативную структуру [13].

Прежде чем переходить к изложению другой точки зрения на возможную комплексификацию инвариантов конечного порядка - мы будем говорить о 
$J$-инвариантах плоских кривых, - сформулируем требования, которым такая комплексификация должна удовлетворять:

- комплексифицированная теория должна описывать когомологии некоторого пространства отображений;

- эти когомологии должны выражаться в терминах дискриминанта в пространстве отображений, стратифицированного в соответствии с вырождениями;

- описание стратов дискриминанта должно формулироваться в терминах некоторых комбинаторных объектов («комплексифицированных хордовых диаграмм»);

- с комплексифицированными хордовыми диаграммами должны быть связаны некоторые алгебраические структуры вроде 4-членного соотношения, алгебры Хопфа, порожденной диаграммами, и т.д.

Если интерпретировать окружность как единичную сферу в двумерной вещественной плоскости, то ее естественным комплексным аналогом служит трехмерная сфера $S^{3}$ - единичная сфера в двумерном комплексном пространстве. При таком соответствии хорда, т. е. 0-мерная сфера $S^{0} \subset S^{1}$, переходит в окружность $S^{1} \subset S^{3}$, а $\mathbb{Z} / 2 \mathbb{Z}$-оснащение хорды заменяется на $\mathbb{Z}$-оснащение окружности. (Напомним, что, согласно Арнольду, комплексификацией группы $\mathbb{Z} / 2 \mathbb{Z}=$ $\pi_{0}\left(S^{0}\right)$ является группа $\mathbb{Z}=\pi_{1}\left(S^{1}\right)$.) Таким образом, комплексифицированной оснащенной хордовой диаграммой можно считать оснащенное зацепление в трехмерной сфере.

Хорошо известно (см., например, [10]), что оснащенному зацеплению $c$ в $S^{3}$ можно сопоставить 4-мерное многообразие с краем, которое мы будем обозначать через $S(c)$. Это многообразие строится следующим образом. Заклеим сферу 4-мерным диском $D^{4}$ и к границе получившегося шара вдоль каждого оснащенного узла приклеим ручку индекса 2 в соответствии с оснащением узла. Отметим, что любое 3-мерное многообразие может быть получено как граница некоторого 4-мерного многообразия вида $S(c)$.

Каждой комплексифицированной хорде (т.е. оснащенному узлу) в $c$ можно сопоставить двумерную сферу в $S(c)$, которая строится так: нужно затянуть узел двумерным диском в приклеенном к сфере $S^{3}$ шаре $D^{4}$, а в качестве второго диска, заклеивающего этот узел, взять «срединный диск» в приклеенной ручке. Полученный набор 2-циклов образует базис во вторых гомологиях $H_{2}(S(c), \mathbb{Z})$ многообразия $S(c)$. Если занумеровать компоненты зацепления $c$ произвольным образом, то индексы пересечения соответствующих им циклов образуют симметричную целочисленную квадратную матрицу, которая является не чем иным, как матрицей индексов зацепления компонент зацепления. По диагонали этой матрицы стоят индексы самозацеплений, которые для оснащенных узлов корректно определены. Эта матрица является комплексным аналогом матрицы примыканий графа пересечений оснащенной хордовой диаграммы. Отметим, что в отличие от вещественного случая, где такая матрица должна подчиняться некоторым ограничениям, в комплексном варианте для любой симметричной целочисленной матрицы существует оснащенное зацепление, индексы зацепления компонент которого образуют такую матрицу.

Первое и второе движение Васильева также имеют естественные комплексные аналоги. Каждое из этих движений определяется парой компонент зацепления (в случае второго движения упорядоченной). Аналогом первого движения 
Васильева служит проведение одной компоненты зацепления через другую, меняющее индекс их зацепления на 1. Аналогом второго является второе двиюение Kирби, которое состоит в протягивании ручки $A$ многообразия $S(c)$ вдоль ручки $B$. В результате второго движения Кирби базис $A, B, C, \ldots$ во вторых гомологиях многообразия $S(c)$ заменяется на базис $A+B, B, C, \ldots$ Таким образом, для комплексифицированных хордовых диаграмм можно написать 4-членное соотношение, однако его смысл остается непроясненным.

Комплексифицированные хордовые диаграммы допускают естественные умножение и коумножение. Произведением двух оснащенных зацеплений в $S^{3}$ служит оснащенное зацепление, представленное связной суммой двух экземпляров трехмерной сферы. Ясно, что такое произведение корректно определено и коммутативно. Копроизведение оснащенного зацепления имеет вид

$$
\mu(c)=\sum c_{I} \otimes c_{J},
$$

где суммирование ведется по всем упорядоченным разбиениям множества компонент зацепления $c$ на два непересекающихся подмножества $I$ и $J$, а через $c_{I}$ обозначено подзацепление зацепления $c$, образованное хордами из подмножества $I$. Введенные умножение и коумножение позволяют превратить векторное пространство, порожденное оснащенными зацеплениями и профакторизованное по 4-членным соотношениям, в алгебру Хопфа.

Самым главным непроясненным фрагментом картины комплексифицированных $J$-инвариантов конечного порядка остается пространство отображений, за которые эти инварианты отвечают. Ясно, что речь идет о каком-то классе отображений трехмерной сферы $S^{3}$ в пятимерную сферу $S^{5}$. Образ трехмерной сферы при общем отображении такого вида имеет лишь трансверсальные самопересечения, прообразы которых образуют гауссову диаграмму отображения. Интересующий нас класс отображений должен быть подобран так, чтобы вырожденные отображения этого класса описывались комплексифицированными оснащенными хордовыми диаграммами. Возможным кандидатом на эту роль служат голоморфные отображения четырехмерного диска $D^{4}$, рассматриваемого как единичный шар в $\mathbb{C}^{2}$, в трехмерное комплексное пространство $\mathbb{C}^{3}$ (мы ограничиваем такое отображение на границу диска и проектируем его образ в единичную сферу в $\left.\mathbb{C}^{3}\right)$.

\section{ЛитеРАТУРА}

1. Arnold V. I. Topological Invariants of Plane Curves and Caustics. Univ. Lecture Ser., Vol. 5, Amer. Math. Soc., Providence, RI, 1994.

2. Arnold V.I. Plane curves, their invariants, perestroikas and classifications. In: Singularities and Bifurcations, Adv. Soviet Math., Vol. 21, Amer. Math. Soc., Providence, RI, 1994, pp. 33-91.

3. Arnold V. I. Symplectization, complexification and mathematical trinities. In: Fields Inst. Commun., Vol. 24, Amer. Math. Soc., Providence, RI, 1999, pp. 23-37.

4. Bar-Natan D. On the Vassiliev knot invariants. Topology, 34, 423-472 (1995).

5. Beck I. Cycle decompositions by transpositions. J. Combin. Theory Ser. A, 23, 198207 (1977).

6. Fenn R., Taylor P. Introducing doodles. In: Topology of Low-Dimensional Manifolds, R. Fenn ed., Lecture Notes in Math., Vol. 722, Springer-Verlag, Heidelberg, 1977, pp. 37-43. 
7. Goryunov V. Vassiliev type invariants in Arnold's $J^{+}$-theory of plane curves without direct self-tangencies. Topology, 37, 603-620 (1998).

8. Hill J. W. Vassiliev-type invariants of plane fronts without dangerous self-tangencies. C. R. Acad. Sci. Paris Sér. Math., 324, No. 5, 537-542 (1997).

9. Казарян М. Э. Инварианты первого порядка типа странности для плоских кривых. Труды МИРАН, 221, 213-224 (1998).

10. Kirby R. A calculus of framed links in $S^{3}$. Invent. Math., 45, 35-56 (1978).

11. Khesin B., Rosly A. Symplectic geometry on moduli spaces of holomorphic bundles over complex surfaces. In: Fields Inst. Commun., Vol. 24, Amer. Math. Soc., Providence, RI, 1999, pp. 311-323.

12. Lando $S$. On a Hopf algebra in graph theory. J. Combin. Theory Ser. B, 80, 104$121(2000)$.

13. Losev A., Manin Yu. New moduli spaces of pointed curves and pencils of flat connections. Michigan Math. J., 48, 443-472 (2000).

14. Mellor $B$. A few weight systems arising from intersection graphs. Michigan Math. J., 51, No. 3, 509-536 (2003).

15. Moran $G$. Chords in a circle and a linear algebra over GF(2). J. Combin. Theory Ser. A, 37, 239-247 (1984).

16. Прасолов В. В., Сосинский А. Б. Узлы, зацепления, косы и трехмерные многообразия. МЦНМО, М., 1997.

17. Soboleva E. Vassiliev knot invariants coming from Lie algebras and 4-invariants. J. Knot Theory Ramifications, 10, 161-169 (2001).

18. Vassiliev V. A. Invariants of ornaments. In: Singularities and Bifurcations, Adv. Soviet Math., Vol. 21, Amer. Math. Soc., Providence, RI, 1994, pp. 225-262.

19. Васильев B. A. Топология дополнений к дискриминантам. Фазис, М., 1998.

20. Vassiliev $V . A$. On finite order invariants of triple point free plane curves. In: Differential Topology, Infinite-Dimensional Lie Algebras, and Applications, Amer. Math. Soc. Transl. Ser. 2, Vol. 194, 1999, pp. 275-300.

Лаборатория Понселе

Независимого московского университета

Институт системных исследований РАН

e-mail: lando@mccme.ru
Поступило в редакцию 10 сентября 2004 г. 